\title{
On the Energetics of Bound Charge-Transfer States in Organic Photovoltaics
}

\author{
Jiangbin Zhang ${ }^{l}$, Andreas C. Jakowetz ${ }^{1}$, Guangru Li ${ }^{l}$, Dawei Di ${ }^{l}$, S. Matthew Menke ${ }^{l}$, \\ Akshay Rao ${ }^{1}$, Richard H. Friend ${ }^{1}$, Artem A. Bakulin ${ }^{1,2 *}$ \\ ${ }^{1}$ Cavendish Laboratory, University of Cambridge, JJ Thomson Avenue, Cambridge CB3 \\ OHE, United Kingdom; \\ ${ }^{2}$ Department of Chemistry, Imperial College London, London SW7 2AZ, United Kingdom; \\ *a.bakulin@imperial.ac.uk
}

\section{KEYWORDS:}

Organic Photovoltaics, Binding Energy, Activation Energy, Arrhenius Plot, Driving Energy, Charge Generation, Pump-Push Photocurrent Spectroscopy, Charge Delocalisation, Device Characterization, Polymer, Fullerene, Ultrafast Spectroscopy; 


\begin{abstract}
A comprehensive understanding of the charge generation mechanism in organic solar cells is critical for further improvement of device performance. Currently, the origin and magnitude of the Coulombic binding energy of the charge-transfer state (CTS), an intermediate state which is fundamental for the charge separation process, are still under debate. Here, we propose a new approach for determining the dissociation energy of localised CTSs for a range of devices with different alignments of molecular energy levels (tuned by chemical modifications of fullerene) and disorder (adjusted by the blend composition) using temperature-dependent pump-push photocurrent spectroscopy. We observe that the dissociation of localised CTSs from initial excitation is a temperature-dependent process, and we determined the binding energy of these CTSs by measuring a single activation energy over a wide temperature range. We propose a simple qualitative picture to explain the observation, based on the split between the bound CTSs and free charges. In all the material systems studied here, the activation energy falls within the range of $90 \pm 50 \mathrm{meV}$ (corresponding to $\sim 1 \mathrm{~nm}$ separation of an electron-hole pair). Surprisingly, the binding energy does not depend on the material composition or the driving energy $(\sim 150 \mathrm{meV}$ variation) for charge separation. In contrast, the number of formed bound states and their following recombination dynamics are material- and nanomorphology-sensitive. Such observations in the studied benchmark polymer:fullerene systems reveal unexpected similarities in the energetics of CTSs formed in different electronic environments. This makes our results of general importance for understanding the photophysics at the heterojunction interface and for further development of organic photovoltaics.
\end{abstract}




\section{Introduction}

Organic photovoltaic cells (OPVs) can efficiently harvest solar energy using two electronically dissimilar organic semiconductor materials - namely electron donor and acceptor. Among the best devices, mainly achieved by morphology optimization, these materials may partially segregate to form an intercalated molecular network architecture known as bulk heterojunction (BHJ). ${ }^{1,2}$ In BHJ devices, generation of free carriers begins with the absorption of a photon by the donor, usually a polymer, or the acceptor, often a fullerene. This light absorption generates a strongly bound intramolecular electron-hole (e-h) pair, called a singlet exciton. The energetic difference between the molecular orbitals of the donor and the acceptor provides the driving force for the dissociation of the singlet exciton into a pair of charge carriers - a hole on the donor and an electron on the acceptor. This initial process is usually addressed as charge transfer (CT) (electron transfer ${ }^{3}$ or hole transfer ${ }^{4}$ ) and the resultant states are often called charge transfer states (CTSs) - an electron-hole pair separated across the donor-acceptor (D-A) heterojunction. In general, the electron and the hole in the CTS are expected to be strongly bound together by a relatively large Coulomb interaction of up to $350 \mathrm{meV}{ }^{5}$ However, in efficient OPV devices, most CTSs subsequently go through a long-range $(\sim 4 \mathrm{~nm})$ charge separation $(\mathrm{CS})$ process to form free mobile carriers (separated-charge states, SC states), while some stay bound at the interface and subsequently recombine before collection at the electrodes.

While this phenomenological picture of OPV operation is broadly accepted, the particular molecular-level mechanisms of CT, long-range CS and recombination in BHJs of organic semiconductor materials are still subjects of discussion. ${ }^{5-14}$ Apart from the simple electrostatic attraction between carriers discussed above, the effects of excess carrier energy, delocalisation ${ }^{15-24}$ and entropy contribution ${ }^{25-29}$ are proposed to control the CS process both in fullerene-based and non-fullerene systems. The interplay between these contributions is at the core of the ongoing debate. For example, even though close-to-unity internal quantum efficiency (IQE) is achieved in some material systems with high driving energy ${ }^{30}$ (roughly the offset between the lowest unoccupied molecular orbitals (LUMO) for electron transfer), it is still unclear whether there is a fundamental limitation for IQE in material systems with low driving energy. Another aspect is the importance of hot CTSs (possessing excess energy above the lowest CTS) versus the relaxed 'cold' CTSs. Critical evidence for supporting the importance of cold CTSs is the similar IQE performances in OPV devices under abovebandgap and sub-bandgap excitations. ${ }^{31,32}$ At the same time, ultrafast spectroscopy 
experiments indicate clear effects of hot or delocalised CTSs on long- and short-range CS. $^{21,22,33,34}$ Still, the direct evidence to support the importance of hot CTSs in efficient operating devices is lacking. We also note that, in line with early studies of morphological effects on solar cell performance ${ }^{35-39}$, recent works emphasise the importance of morphology for the photophysics of CT states. ${ }^{40,41}$

To build a unified model of charge generation, many groups have focused on the fundamental properties of CTSs and addressed different aspects of photophysics of CTSs, including energetic structures, relaxation rates and coupling to excitonic and SC states. ${ }^{15,42-51}$ Probably the most fundamental parameter characterizing the localised CTSs is their binding energy, which can be defined as the energy difference between the SC state and the localised CTS. ${ }^{18,52,53}$ For this reason, many different, mostly steady-state, approaches have been applied to determine the binding energy of localised CTSs. ${ }^{42,54-56}$ However, the findings of these studies do not converge to the same value. The most obvious reasons for this are the complexity of measuring the energy levels of SC states, the fast relaxation time of localised CTSs, the influence of internal electric-field and the dispersion of energy levels in the blends with inhomogeneous nanomorphology.

Temperature-dependent ( $\mathrm{T}$-dependent) measurements provide a robust tool to probe material energetics and to achieve further insight into the charge generation mechanism, inspiring numerous studies on the influence of temperature on CS process in various material systems. ${ }^{34,57-65}$ The majority of these studies aim to relate the T-dependent variation of device parameters, including mainly short-circuit current $\left(J_{s c}\right)$ and open-circuit voltage $\left(V_{o c}\right)^{25,44,66-68}$ with the charge generation process. The results from these measurements have been mainly rationalised in the framework of the Onsager-Braun model and its modifications. We note that Onsager model (which regards CT states as precursor states for charge generation) has many limitations and it fails to explain, for example, energetic disorder and BHJ morphology dependence. Temperature, together with the external electric field, is considered to be a most critical parameter in the dissociation efficiency of CTSs (precursors for free charges). ${ }^{69}$ Though these models are able to reproduce some experimental results, the fitting parameters in the dissociation efficiency formula are not always consistent with other measurements or sometimes not physically reasonable. ${ }^{9}$ The reason for this, as some authors have pointed out, is that the steady-state photocurrent arises from multiple T-dependent processes including (CT) exciton dissociation, charge transport, and bimolecular recombination. ${ }^{25,69}$ This prevents steady-state measurements from selectively addressing the energetics of CTSs and the early- 
time non-equilibrium carrier dynamics. The issue of T-dependent extraction and bimolecular recombination (BR) can potentially be sorted out by considering that all photogenerated e-h pairs recombine in the open-circuit condition. In such a case, $V_{o c}$ might be less affected by the charge transport process, allowing for the exclusive focus on the charge separation process at the D-A interface. Recently, Gao et $a l^{25}$ explained the deviation from linear $V_{o c}-T$ relationship at low $T$ due to ineffective geminate dissociation. To summarise, steady-state measurements, in and by itself, have two fundamental weaknesses: (i) The processes of (CT) exciton dissociation, charge recombination and charge extraction all contribute to device performance but may have different dependence on temperature. ${ }^{31,64}$ (ii) Geminate recombination (GR) and BR cannot be easily disentangled when the integrated photocurrent is solely measured.

The above issues can be solved by looking at early-time charge dynamics, or using techniques that are selective to bound CTSs. Previous time-resolved studies on CTSs mostly addressed CTS emission. For example, time-resolved photoluminescence (TR-PL) using transient grating ${ }^{70}$ or streak camera ${ }^{65}$ can measure radiative recombination of CTSs. The recent T-dependent TR-PL measurements revealed quite different dynamics of CTSs in two benchmark material systems ${ }^{59,60,71}$, supposedly coming from the differences in non-radiative CTS recombination. Using transient absorption spectroscopy, Barker et al. observed tunneling recombination by freezing carrier movement at cryogenic temperature and measured the e-h separation distance $\mathrm{e}^{72}$. Overall, previous works provide many insightful case studies of CT relaxation and report a large range of binding energies of CTSs (0-350 $\mathrm{meV})^{5,55,72,73}$, with the highest values close to the binding energies of the singlet excitons. However, a systematic and selective investigation of the CTS photophysics in a working device, which correlates the device performance with the binding energy of localised CTSs, is still missing.

In this paper, we perform temperature-dependent ultrafast pump-push photocurrent (PPP) spectroscopy on a range of material systems based on a benchmark polymer poly[2-methoxy5-(3,7-dimethyloctyloxy)-1,4-phenylen]-alt-(vinylene) (MDMO-PPV) and a range of fullerene derivatives. PPP is a device-based technique which selectively monitors the formation and recombination of bound CTSs on the ultrafast (ps) time-scale. These bound CTSs are a subset of the total localized CTSs. They cannot escape the interface and as a consequence experience only geminate recombination. T-dependent PPP offers a possibility of overcoming the drawbacks of other methods by specifically targeting the long-range CS of bound CTSs at the interface. With three different fullerene derivatives, the role of the driving 
energy as well as material morphology is investigated. We first compare the device performance, then we introduce the PPP technique by elucidating the dynamics of bound CTSs at room temperature. Our low-temperature measurements show that the yield of bound CTSs is temperature-dependent. Using the Arrhenius model, we extract a single activation energy for the CTS dissociation which we associate with the binding energy of the localised CT excitons. A simple picture with the direct branching between bound CTSs and free charges can qualitatively explain the observed trend. The average activation energy of $90 \pm$ $50 \mathrm{meV}$ indicates the energetic similarities of bound CTSs regardless of driving energy for charge separation (as provided by donor-acceptor energetic offset) and material composition, in sharp contrast with the obvious trend in device efficiencies. With a simple electrostatic calculation, we relate the activation energy of the bound CTSs to an e-h separation of around $1 \mathrm{~nm}$. We emphasise that it is not the energy of CTSs that matters; it is the population density of the localised states for bound carriers that controls early charge separation and recombination. In devices with a good morphology, most states are delocalised and very few bound CTSs form at the interface, leading to a better device performance. Our result points to the importance of minimizing the number of bound states by morphological control rather than by lowering the dissociation energy of bound CTSs.

\section{Experimental Methods}

2.1 Materials and Devices. MDMO-PPV was adopted as the donor material which was purchased from Merck (lisicon PDO-121). Three different fullerene derivatives were used as acceptors: Phenyl-C61-butyric acid methyl esters mono- $\mathrm{PC}_{61} \mathrm{BM}(\mathrm{mPCBM})$, bis- $\mathrm{PC}_{61} \mathrm{BM}$ (bPCBM), tris- $\mathrm{PC}_{61} \mathrm{BM}(\mathrm{tPCBM})$ from Solenne BV.

ITO-coated glass substrates were cleaned by sonication in acetone and isopropyl alcohol, before being exposed to $\mathrm{O}_{2}$ plasma at $250 \mathrm{~W}$ for $10 \mathrm{~min}$. PEDOT:PSS aqueous solution (P VP Al 4083, Heraeus) was filtered with $0.45 \mu \mathrm{m}$ hydrophilic filter, before being spin-coated on the substrate at $5000 \mathrm{RPM}$. The substrate was dried at $120{ }^{\circ} \mathrm{C}$ under nitrogen atmosphere for $30 \mathrm{~min}$. The subsequent processes were performed in a nitrogen glovebox $\left(\mathrm{O}_{2}<5 \mathrm{ppm}\right.$, $\left.\mathrm{H}_{2} \mathrm{O}<1 \mathrm{ppm}\right)$. Solution with different blend ratios (1:1, 1:2 and 1:4 weight ratios) of MDMO-PPV:fullerene derivatives were prepared in different concentrations in orthodichlorobenzene $\left(10 \mathrm{mg} / \mathrm{ml}, 12.5 \mathrm{mg} / \mathrm{ml}\right.$ and $15 \mathrm{mg} / \mathrm{ml}$ respectively) by stirring at $50{ }^{\circ} \mathrm{C}$. The solution was then spin-coated onto the PEDOT:PSS layer at 1500 RPM for 40 seconds followed by 2000 RPM for 20 seconds. ${ }^{74}$ An $80 \mathrm{~nm}$ layer of aluminium was deposited by 
thermal evaporation method under high vacuum $\left(\sim 10^{-6} \mathrm{mbar}\right)$. The device current-voltage curves were measured using a Keithley 2635 source-meter under the illumination of an Oriel 81160-1000 solar simulator. We typically measure 3 pixels to ensure that the device performance is reproducible.

\subsection{Pump-Push Photocurrent (PPP) Spectroscopy}

In the PPP experiment, a regenerative $1 \mathrm{kHz}$ Ti:Sapphire amplifier system (Spectra Physics, Solstice) generated ultrafast $100 \mathrm{fs}$ laser pulses at $800 \mathrm{~nm}$ with the energy of $3.5 \mathrm{~mJ}$ per pulse, which was split into two parts. One part was used to pump a broadband non-linear optical amplifier (NOPA) to produce visible pump pulses with the photon energy of $2.30 \mathrm{eV}$ (540 $\mathrm{nm})$. The other part was used to generate mid-infrared (IR) push pulses $(\sim 2 \mu \mathrm{m}, \sim 0.62 \mathrm{eV})$ by pumping an optical parametric amplifier (TOPAS, Light Conversion). The device (connected to the external circuit outside the cryostat) was situated in a low-temperature cryostat (Optistat CFTM, Oxford Instruments), and was immersed in the helium gas environment during measurements. Temperature inside the cryostat was detected by a sensor of a temperature controller (ITC 503, Oxford Instruments) and controlled by the balance between its heat generation and the flow of helium gas adjusted by a gas-flow controller (VC41, Oxford Instruments).

During the experiment, the devices were measured at the short-circuit condition under different temperatures. Pump pulses (with energy $\sim 1 \mathrm{~nJ}$ per pulse) and push pulses (with energy $\sim 1 \mu \mathrm{J}$ per pulse) were focused onto $\mathrm{a} \sim 1 \mathrm{~mm}^{2}$ spot on the device. The reference photocurrent, $J$, induced by the pump pulse was detected at the laser frequency of $1 \mathrm{kHz}$ by a lock-in amplifier (SRS830, Stanford Research System). The repetition rate of the push pulses was modulated by a mechanical chopper (MC2000, Thorlabs) at $370 \mathrm{~Hz}$ which was synchronised with the lock-in amplifier to detect the push-induced photocurrent, $d J$. We note that the photophysics in the pulse mode excitation may be different from steady-state illumination, or under different fluences. Previously, Marsh et al. ${ }^{75}$ performed detailed analyses on the fluence-dependence of external quantum efficiency (EQE) in P3HT:PCBM (poly(3-hexylthiophene-2,5-diyl, P3HT) devices. In our measurements, very low fluence $\left(\sim 100 \mathrm{~nJ} / \mathrm{cm}^{2}\right)$ and low photocurrent $(<100 \mathrm{nA})$ ensure the device to work in a similar condition as under a standard one-sun illumination. This shows the advantage of PPP over pump-probe spectroscopy to observe the charge dynamics in the working devices under lowfluence excitation. 


\section{Results}

\section{Materials and device performance}

Figures 1 (a) and (b) present the chemical structures and energy levels of the used materials, respectively ${ }^{76}$. The bandgaps of three fullerene derivatives are similar $(\sim 1.7 \mathrm{eV})$, but the highest occupied molecular orbital (HOMO) and the LUMO energy levels are very different. This allows for the investigation of the influence of the driving energy on charge separation process. The film absorption spectra of these materials are shown in Figure 1 (c) and the blend film absorption data are shown in Figure S1. The advantage of MDMO-PPV is that its electronic energy levels have negligible change when the fullerene weight ratio changes. ${ }^{76}$ It is well mixed with fullerenes and forms different morphologies of intercalating polymer/fullerene network in the studied blending ratios (1:1, 1:2 and 1:4).

a

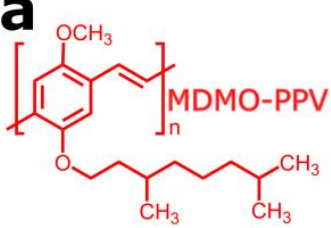
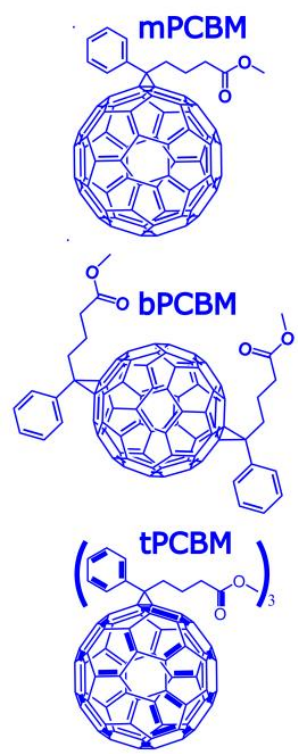

b
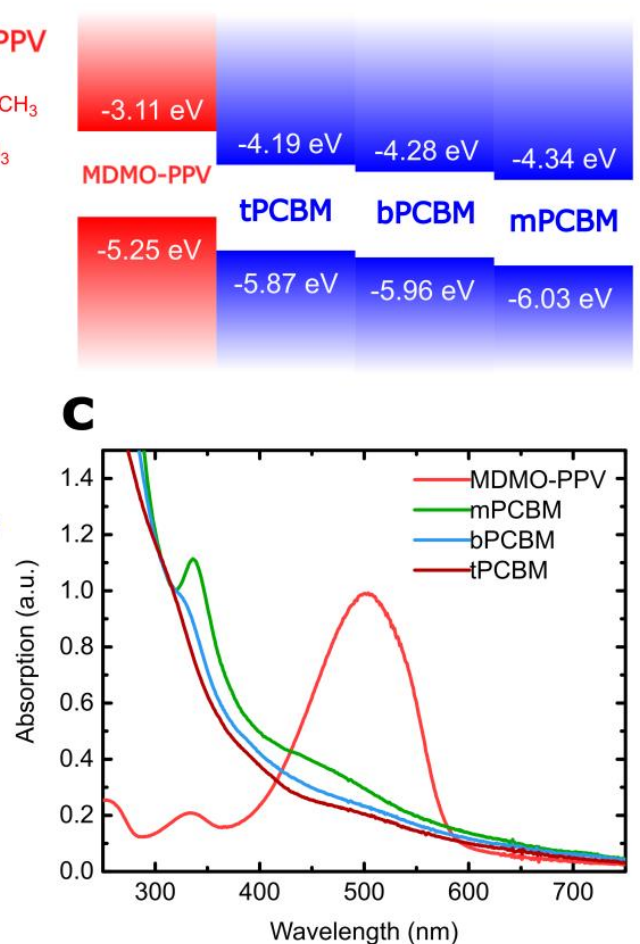

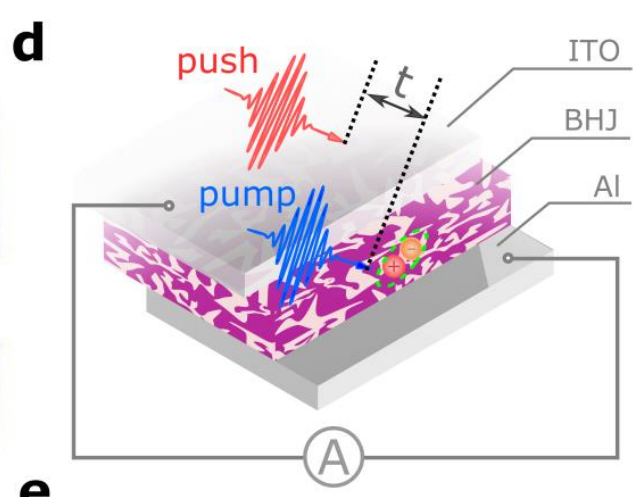

e

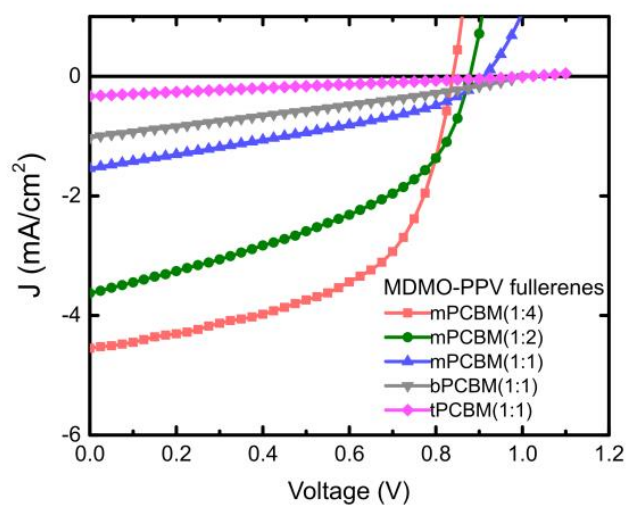

Figure 1. (a) Chemical structures of the donor polymer MDMO-PPV and three fullerene acceptor derivatives: mPCBM, bPCBM and TPCBM. (b) HOMO and LUMO energy levels and (c) Normalised absorption for the materials. (d) Device architecture where the polymer-fullerene blend is sandwiched between the hole transporting layer (PEDOS:PSS) and the aluminium electrode. The device is held at short-circuit during the pump-push measurement (e) J-V curves for MDMO-PPV:mPCBM (blend ratios, 1:1, 1:2 and 1:4), MDMO-PPV:bPCBM (blend ratio, 1:1), and MDMO-PPV:tPCBM) (blend ratio, 1:1) devices under $100 \mathrm{~mW} / \mathrm{cm}^{2}$ illumination at AM1.5G. 
These MDMO-PPV-based solar cells in Figure 1 (d) were fabricated following the procedures described in the experimental section. Typical $J$ - $V$ curves are shown in Figure 1 (e) and the full set of device parameters are summarised in Table S1. From the $J-V$ data, increasing the fullerene loading or decreasing the number of side chains in the fullerene improve the device power conversion efficiency ( $P C E$ ) through improved $J_{s c}$ and fill factor $(F F)$. This trend is in good agreement with the fullerene packing and aggregate size measured by small-/wide-angle X-ray scattering ${ }^{76}$, which indicates the importance of wave-function delocalisation induced by fullerene aggregation. ${ }^{15}$ Previously, transient absorption techniques indicated that the electron transfer time from polymer to fullerene derivatives ranges from 37 fs to $100 \mathrm{fs}$, and a high driving energy is not needed to facilitate this ultrafast CT. Combined with our device data, it is logical to assume that the great differences in $J_{s c}$ and PCE do not originate from the ultrafast $\mathrm{CT}$, but rather from the differences in the subsequent long-range CS, charge transport and extraction processes.
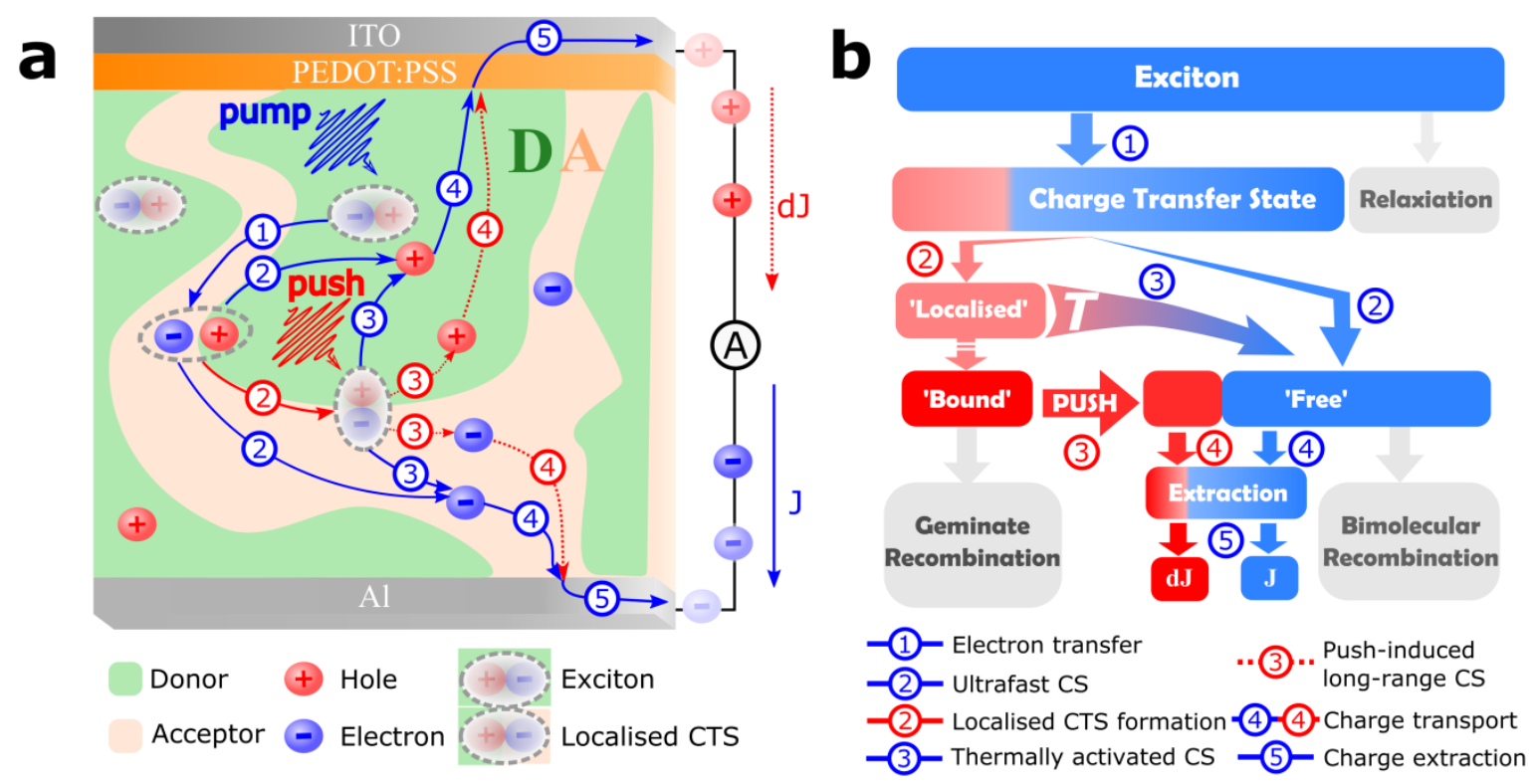

Figure 2. Model of photogeneration processes involved in the PPP experiment in organic solar cells. Without electron acceptors, excitons generated by the pump pulses in the polymer phase recombine into the ground state. In the presence of a good acceptor, the ultrafast CT processes quench singlet excitons before relaxation and form initial CTSs at the D-A interface (process 1). These initial CTSs subsequently branch between 'localised' CTSs and partial 'free' charges (process 2) on sub-ps time scale. Those CTSs thermalise into bound charge pairs can only geminately recombine to the ground state, while some localised CTSs separate into 'free charges' by thermal activation (process 3 , blue) later. With the IR-push pulses, these bound states are re-excited into higher-energy CTSs, accessing delocalised states capable of spontaneously separating into 'free' charges from another channel (process 3, red). Those separated 'free' charges instead interact through bimolecular recombination 
or flow to the electrodes through charge transport in the separated phases (process 4). These additional 'free' charges and previous 'free' charges are extracted as $d J$ and $J$ by external circuits (process 5), respectively. Note that triplet states are not considered in this model.

\section{Room-temperature PPP experiments.}

To study the influence of film morphology and driving energy on charge separation efficiency, it is necessary to separate GR and BR processes which dominate the dynamics on different time scales. To compare specifically the difference in the early-time charge dynamics, we perform pump IR-push photocurrent spectroscopy on these working devices. ${ }^{21}$ The PPP experiment directly measures the formation and recombination of bound CTSs at the interface in real time by detecting the additional photocurrent due to the push pulses which provide additional energy to dissociate these bound states at the D-A interface. Figure 2 further illustrates the relevant electronic processes in the PPP method. The pump pulse at around $550 \mathrm{~nm}$ first generates excitons in the MDMO-PPV donor. With the acceptor, the fullerene efficiently quenches the excitons at the D-A interface by ultrafast electron transfer and form some intermediate CTSs. These CTSs then branch into either localised CTSs or undergo long-range CS to form free charges. Another part of free charges come from the thermally activated charge separation of localised CTSs. The separated total free charges are collected as photocurrent, $J$. The bound CTSs undergo GR, unless the push pulses arrive and excite the bound CTSs to the delocalised band-like states to give them another chance to dissociate. This re-excitation effect is observed as an additional photocurrent $d J$ detected by a lock-in amplifier. The maximal amplitude $d J_{\max } / J$ of the normalised dynamics, $d J / J$, is used to quantify the charge separation efficiency, and is discussed in detail in the next section.

Figure 3 shows the PPP response at room temperature $(300 \mathrm{~K})$ with blend ratios of 1:1 and 1:2. Full dataset at room temperature is included in Figure S2. The background signal observed at negative delays is corrected for, as this background is usually associated with long-lived trapped charges and is not a subject of the current study. Some curves of PPP kinetics without background removal can be found in Figure S3. As trapped charges and states take much longer time ( $\gg 1 \mathrm{ps}$ ) to be observed, the significant increase at time zero must be due to the onset presence of bound CTSs. We find that the lifetime of bound CTSs changes from acceptor to acceptor, but does not strongly depend on blend composition. Also, the devices with higher fullerene loadings consistently have higher CS efficiency in all three fullerene devices, which agrees well with the consideration that the morphology is more 
important for charge generation than the driving force for charge separation provided by donor-acceptor band alignments. ${ }^{76}$

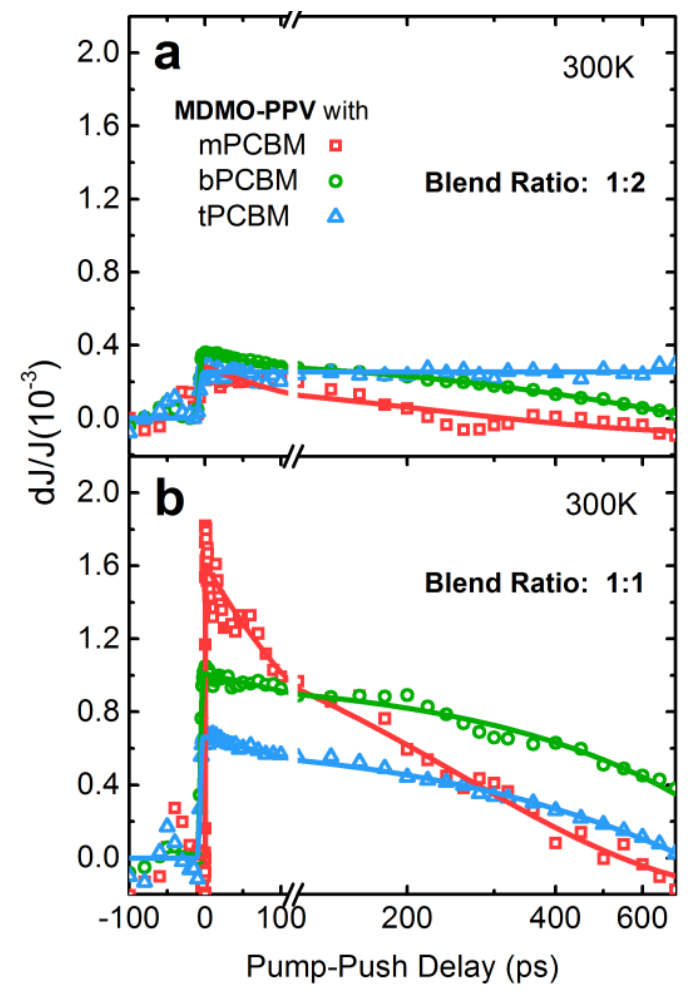

Figure 3. PPP dynamics at room temperature (300 K) of devices with D-A ratios of (a) 1:2 and (b) 1:1. Solid lines are guides for the eye.

\section{Temperature-dependent PPP experiments.}

In Figure 2, we assume that there is an early branching between localised CTSs and direct free charge generation. Direct evidence comes from ultrafast experiments where free charges can be generated just after charge transfer ${ }^{9,18,22,72,76-78}$, and other experiments ${ }^{60,65,78}$ also support this assumption in both $\mathrm{BHJ}^{18,21,77}$ and bilayer ${ }^{79}$ devices. After CT, the initial hot CTSs form. As fast as in $1 \mathrm{ps}^{21}$, either the formation of localised CTSs or generation of free charges via long-range CS may take place. ${ }^{80}$ Thermal dissociation of localised CTSs also contributes to the photocurrent and are not observed in PPP, thus are not addressed as 'bound'. The properties of material system define how many of these hot CTSs form bound CTSs, $N_{\text {bound }}(T)$ and how many separate into free charges, $N_{\text {free }}(T)$. Some of these free charges can escape from BR and generate photocurrent $J(T)$, thus

$$
J(T) \propto N_{\text {free }}(T) * \eta_{\text {extract }}(T)
$$


where $\eta_{\text {extract }}(T)$ is the extraction efficiency of free charges to the electrode from charge separation. By absorbing IR push photons, bound CT excitons are excited into higher-lying band-like delocalised states, after which being dissociated into free charges ${ }^{21}$. The additional 'push'-induced photocurrent is extracted and measured with the lock-in amplifier as $d J(T)$. By changing the time delay between the pump and push pulses, we get

$$
d J(T, t) \propto N_{\text {bound }}(T, t) * P_{\text {activation }}(T) * \eta_{\text {extract }}(T)
$$

Here $N_{\text {bound }}(T, t)$ is the number of bound CTSs when the push pulse arrives at time $t$ after the pump pulse, and $P_{\text {activation }}(T)$ is the probability of the bound CTSs to absorb the infrared photons and dissociate into free carriers. The decay of $d J(T, t)$ is assigned to the decay of bound CTSs at the interface with time due to GR. It is important to point out that free charges (either directly generated from excitons or from thermal dissociation of localised CTSs) also absorb the infrared light, but they contribute to the photocurrent even without the push pulses. Therefore, there is no net contribution of free carriers to the modulated photocurrent. Both the generations of $J$ and $d J$ are mediated by the similar charge transport and extraction processes after CS. Consequently, we use the same charge extraction efficiency $\eta_{\text {extract }}(T)$ (including charge transport process) for Equation (1) and Equation (2).

Considering that the push-induced re-excitation of charges brings the system into a highly non-equilibrium state, we assume $P_{\text {activation }}(T)$ is a constant at different temperature, and thus we have

$$
\frac{d J(T, t)}{J(T)} \propto \frac{N_{\text {bound }}(T, t)}{N_{\text {free }}(T)}
$$

The PPP transients sometimes show additional fast component within the pump and push overlap ( $t<200 \mathrm{fs}$ ). This can be caused by multiphoton non-resonant effects like sumfrequency generation and are irrelevant to the process of CS. If this effect is pronounced, we can quantify $d J_{\max }(T)$ after a few picosecond delay rather than using the maximal amplitude,

$$
\frac{J(T)}{d J_{\max }(T)} \propto \frac{N_{\text {free }}}{N_{\text {bound }}}(T) \sim \eta_{C S}(T)
$$

where $\eta_{C S}$ is the charge separation efficiency at temperature $T$. This parameter measured from PPP experiment is proportional to thebranching between bound CTSs and free charges, which quantifies the CS efficiency at the interface. Since the temperature dependence of the 
extraction process is cancelled out, we can more clearly monitor the effect of temperature on long-range CS process, and directly observe the dynamics and yield of the bound carriers.

To analyse the temperature-dependent behaviour, we assume that there is a single activation energy needed for the thermally activated CS process to occur. Arrhenius plots are widely used to characterise temperature-dependent measurements and the activation energy can be extracted to phenomenologically represent the barrier of some chemical or electronic processes. The full set of data is in Figure S4. Here, we take a MDMO-PPV:bPCBM device with 1:1 blend ratio as an example. Figure 4 shows the result of temperature-dependent steady-state photocurrent $J(T)$ and PPP spectroscopy at temperatures from $160 \mathrm{~K}$ to $300 \mathrm{~K}$. The blue curve in Figure 4 (b) shows the change of photocurrent measured at different temperatures. The constant decrease agrees with previous results ${ }^{69}$ and might be caused by the variation of $N_{\text {free }}(T)$ or $\eta_{\text {extract }}(T)$ from Equation (1). The Arrhenius plot in Figure 4 (c) (blue curve) does not show a single activation energy from $300 \mathrm{~K}$, which confirms that a direct photocurrent response represents a convolution of multiple T-dependent processes.
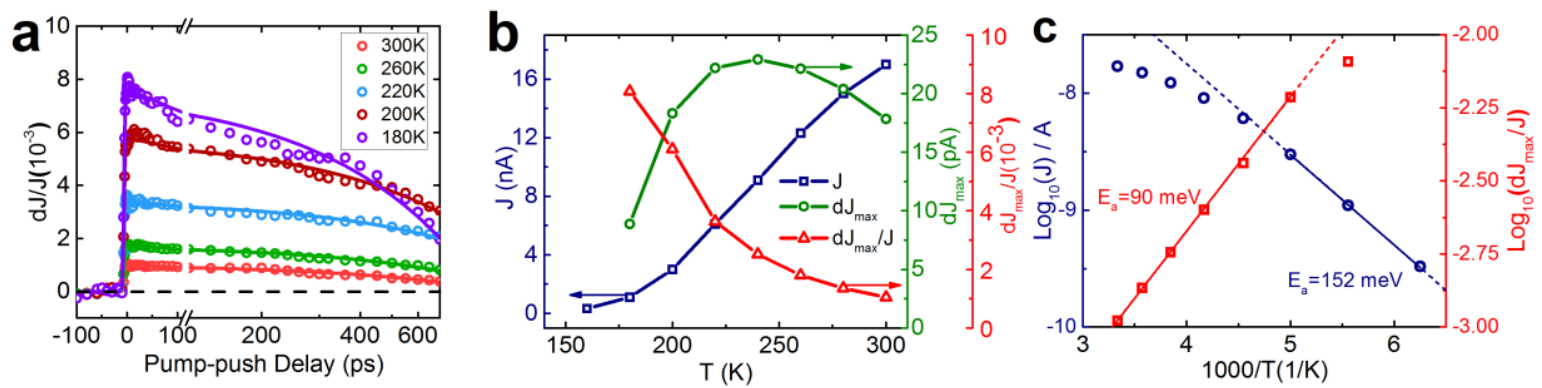

Figure 4. Temperature-dependent PPP response of MDMO-PPV:bPCBM (1:1) from $160 \mathrm{~K}$ to $300 \mathrm{~K}$. (a) PPP dynamics on the sub-ns time scale with a guideline at $d J / J=0$ for comparison. (b) Photocurrent ( $J$, blue), push-induced photocurrent change $\left(d J_{\max }\right.$, green) and $1 / \eta_{C S}\left(d J_{\max } / J\right.$, red) at different temperatures. (c) Arrhenius plots for $J$ (blue) and $1 / \eta_{C S}$ (red); $\eta_{C S}(T)$ showed an activation energy of $90 \mathrm{meV}$ from $200 \mathrm{~K}$ to $300 \mathrm{~K}$ while the activation energy for $J$ was $152 \mathrm{meV}$ below $200 \mathrm{~K}$. Solid segments show respective linear fits and dashed lines illustrate the extrapolation of the solid lines.

Figure 4 (a) presents the PPP kinetics at different temperatures. At all temperatures, the maximal amplitude is obtained within $1 \mathrm{ps}$, which indicates that exciton diffusion is not a limiting factor for exciton dissociation. ${ }^{80}$ In Figure $4(\mathbf{b})$, the peak at $240 \mathrm{~K}$ in $d J_{\text {max }}$ curve (green curve) can be rationalised as an interplay between a decrease in the number of bound carriers and an increase in $\eta_{\text {extract }}(T)$ at higher temperatures, according to Equation (2). At lower temperatures, the charge mobility determines $d J_{\max }$ and it steadily decreases in the 
range from $240 \mathrm{~K}$ to $160 \mathrm{~K}$. The normalised photocurrent $\left(d J_{\max } / J\right.$, red curve $)$ in Figure 4 (b) effectively provides a correction for the extraction efficiency and shows a drastic increase in the yield of bound carriers at low temperatures. By using Arrhenius analysis as shown in Figure 4 (c), we obtain a single activation energy of $90 \mathrm{meV}$ for $1 / \eta_{C S}(T)$ in the range from $200 \mathrm{~K}$ to $300 \mathrm{~K}$. We emphasise again that this single activation energy is absent in either $J$ or $d J_{\max }$ at higher temperatures due to an interplay between the number of bound CTSs and temperature-dependent charge extraction. Thus, this single activation energy strongly demonstrates the significance of the $\eta_{C S}(T)$ defined in this paper. From this simple picture, we have

$$
\eta_{C S}(T) \propto \frac{N_{\text {free }}}{N_{\text {bound }}}(T) \propto e^{-\frac{E a}{k_{B} T}}
$$

where $E_{a}$ is the activation energy of bound CTSs, $T$ is the temperature and $k_{B}$ is the Boltzmann constant.

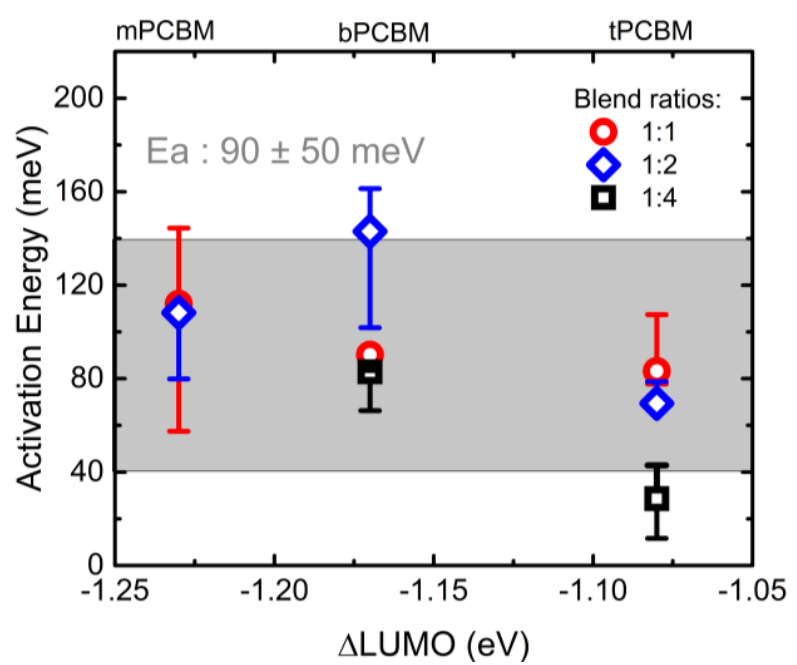

Figure 5. Activation energy as a function of driving energy in the studied blends with different donor: acceptor blend ratios (1:1,1:2 and 1:4). Driving energy is taken roughly as the energy difference between the LUMO energy levels of MDMO-PPV and the respective fullerene derivatives. The uncertainty of the activation energy is obtained from the linear fit of the Arrhenius plot and is described in the Supporting Information.

Figure S4 shows all the pump-push data of the measured devices as well as the Arrhenius graphs with the fitted lines for the activation energy $\left(E_{a}\right)$. The activation energy is summarised in Figure 5. We find that $E_{a}$ does not change much across the whole range of samples $-E_{\mathrm{a}}$ is neither a function of LUMO offset, nor is a function of D-A ratio. The 
average value of all these activation energies is $90 \mathrm{meV}$ and the horizontal dark grey line shows the energy level in the graph. To our surprise, there is no obvious correlation between the material composition or driving energy with the change of activation energy. We assume an uncertainty of $50 \mathrm{meV}$ higher or lower than the $90 \mathrm{meV}$ "median value" to include all of our data ranging from $40 \mathrm{meV}$ to $140 \mathrm{meV}$, shown as grey area in Figure 5. We note that 150 $\mathrm{meV}$ is a narrow range to tune the driving energy. However, a number of works have shown that even modest variations in the driving energy can lead to a substantial change in photophysics. ${ }^{5,81}$

Table 1 Transition temperature in the Arrhenius plots for $d J_{\max } / J$ in different devices.

\begin{tabular}{|c|c|c|c|}
\hline Acceptor & 1:1 & $1: 2$ & $1: 4$ \\
\hline IPCBM & $240 \pm 10 \mathrm{~K}$ & $230 \pm 10 \mathrm{~K}$ & \\
\hline bPCBM & $190 \pm 10 \mathrm{~K}$ & $230 \pm 10 \mathrm{~K}$ & $230 \pm 10 \mathrm{~K}$ \\
\hline tPCBM & $172.5 \pm 7.5 \mathrm{~K}$ & $205 \pm 15 \mathrm{~K}$ & $247.5 \pm 7.5 \mathrm{~K}$ \\
\hline
\end{tabular}

From the Arrhenius plot, there is a transition temperature in $\eta_{C S}(T)$, where the Arrhenius model does not apply anymore. This transition temperature is quite different across devices, as summarised in Table 1. The deviation from linearity might come from many reasons. We exclude the possibility that the absorption cross section of CTSs changes dramatically within this temperature range. The transition temperature correlates nicely with the device efficiency data: a higher device efficiency corresponds to a higher transition temperature. At higher temperatures, the push pulses could efficiently separate the bound CTSs and contribute more significantly to the photocurrent, while at lower temperatures, even though the CTSs may already be separated at the first place, the separated charges may experience a higher level of local disorder which reduces the mobility, leading to more severe BR. This peak temperature might be regarded as a parameter for characterising the influence of BR on the device performance.

\section{Discussion}

Our results demonstrate that the dissociation of localised CTSs is a temperature-dependent process in the material systems studied. The extracted activation energy is one of the 
fundamental parameters describing the energetic landscape at the heterojunction interface for CS after photoexcitation. The observed single activation energy value supports the assumptions which we have made during the analyses, including (i) the exciton dissociation in our study is not a temperature-dependent process ${ }^{25}$, (ii) the activation of the bound CTSs by a push pulse is not temperature-dependent, and (iii) branching between localised CTSs and those directly contributing to free carriers occurs on the sub-ps timescale.

Here, we note that the push pulses acting on the free charges, followed by the fast thermalisation, might enhance local charge mobility. However, this does not influence the measureable quantities ( $d J$ and $J$ ) in PPP for two reasons: (i) As the free charge is excited to a higher state only once and for just $\sim 0.1 \mathrm{ps}^{21}$, the gain in diffusion is probably no more than 5 $\mathrm{nm}$ considering the disordered electronic structure away from the interface ${ }^{23}$. Therefore the effect caused by enhanced mobility should be negligible. (ii) As the following charge collection process is very slow ( $\sim \mathrm{ms}$ for $1 \mathrm{kHz}$ experimental setup we use), the enhanced mobility would just slightly change the total time of charge collection $(<1 \mathrm{~ms})$, but not the total number of extractable free carriers.

The observed activation energy of $90 \mathrm{meV}$ for localised CTSs corresponds to a $\sim 1 \mathrm{~nm}$ separation of the e-h pair if the simple electrostatic attraction model is used. In our calculation, we evaluate the binding energy using a classical electrostatic model where the energy needed to separate an electron from a hole is proportional to $1 / r$ ( $r$ is the e-h separation distance and the energy at the infinite distance is defined as zero). ${ }^{18,52}$ As numerous experimental results indicate an e-h separation of $4 \mathrm{~nm}$ for mobile carriers ${ }^{18,72}$, we estimate the binding energy of free carriers to be equivalent to the thermal energy at room temperature $(\sim 25 \mathrm{meV})$. For bound CTSs, the e-h separation measured from tunnelling recombination is $0.5-1 \mathrm{~nm}^{72}$, which corresponds to $75-175 \mathrm{meV}$ and agrees well with the binding energy $(90 \pm 50 \mathrm{meV})$ we measured. The average binding energy of $90 \mathrm{meV}$ gives a reasonable $\sim 1 \mathrm{~nm}(0.87 \mathrm{~nm})$ e-h separation for bound CTSs. We further estimate the smallest activation energy of $28 \mathrm{meV}$ measured in the tPCBM device with a D-A ratio of 1:4, corresponding to an e-h separation of $1.9 \pm 0.3 \mathrm{~nm}$, assuming $20 \%$ variation in the dielectric constant across the samples in different fullerene loadings. This larger e-h separation might be connected to CT delocalisation which lowers the binding energy of the bound CTSs and facilitates efficient charge separation. 


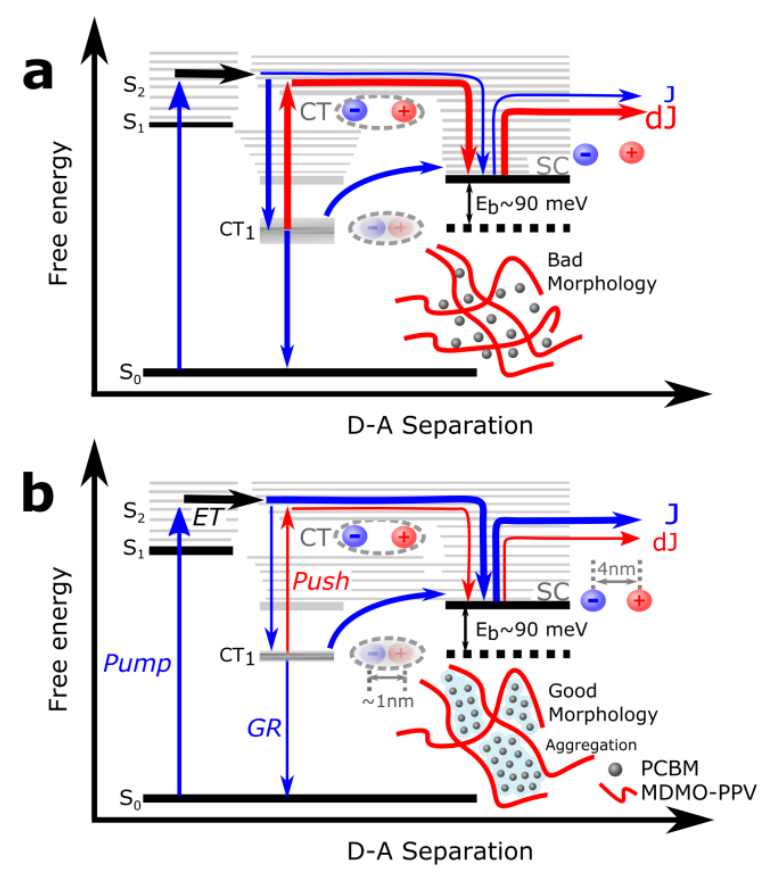

Figure 6. State energy diagram describing the effect of fullerene aggregation on free charge generation. Pump-induced charge dynamics is shown in blue and push-affected dynamics in red. In both situations, e-h separation occurs at a separation of $4 \mathrm{~nm}$. The binding energy of $\sim 90 \mathrm{meV}$ corresponds to $\sim 1 \mathrm{~nm}(0.87 \mathrm{~nm})$ of e-h separation. The presence of fullerene aggregation in (b) promotes delocalised CTSs and fewer bound CTSs are formed, compared with relatively bad morphology in (a). The push pulse promotes the bound CTSs to delocalised states which can then separate into free charges spontaneously.

Surprisingly, the binding energy of bound CTSs does not show a clear correlation with material composition or driving energy despite the huge difference in device performance (See Table S1). For fullerene derivatives, this might be due to a reduced density of side chains that reduces the level of disorder and increases the electron mobility. This can greatly influence the BR process, affecting $F F$ and $J_{s c}$. Figure $\mathbf{S 1}$ shows that the higher fullerene loading increases the charge separation efficiency. This points to the importance of fullerene aggregation in efficient charge generation. Morphology influences the degree of delocalisation of the CTSs and determines the number of bound carriers at the interface, while the energetic difference between the bound CTSs and the SC states is similar, see Figure 5. For fullerene derivatives with different driving energies at the interface, the energy levels of the bound CTSs probably change as there is a linear relationship between $V_{o c}$ and the energy levels of the CTSs. However, the binding energies of bound CTSs are still similar. We point out that this $90 \mathrm{meV}$ binding energy ( 1 nm e-h separation) of the localised CTSs measured in benchmark polymer:fullerene systems with a range of driving energies and film 
morphologies should be quite typical for organic solar cells, and it indicates the unexpected similarities of the energetics between bound CTSs in OPVs.

In the analysis we use early-time $d J / J$ because this excludes the contribution of temperaturedependent non-radiative recombination process. PPP measurements are not sensitive to the particular dynamics of temperature activation, but only to the activation efficiency. The further investigation of combining temperature-dependent time-resolved PL and PPP might give insight into the CTS radiative and non-radiative recombination as well as its dissociation. Apart from $\eta_{C S}$, the kinetics of $d J / J$ may contain information about GR and other processes involved in the early time window (around $1 \mathrm{~ns}$ in our data). In our model, the decay of bound CTSs is only caused by geminate recombination, and not related to the rate of thermal dissociation into free carriers. The localised states that thermally separate are likely to spend different 'activation' time at the interface at different temperatures, but these states, as we mention before, are not observable in the kinetics of $d J / J$. Therefore, the initial amplitude of pump-push signal is sensitive to the balance between the number of bound and free carriers, but not to the time-evolution of the whole localised states.

Our results agree well with the current design rules of efficient OPVs. Improved material systems are expected to have reduced LUMO offsets (for smaller voltage loss), charge delocalisation (for efficient charge separation) and higher charge mobilities (for better charge collection) to achieve efficient solar cell operation. This improvement is a challenge for fullerene-based solar cells due to the limited choice of acceptor properties. Non-fullerene acceptors offer more synthetic flexibility, providing the possibility to simultaneously fulfil these design criteria, leading to OPVs with low voltage loss, high photocurrent and high fill factor. Recent research has shown some promising development of non-fullerene organic solar cells which satisfy our design considerations ${ }^{82-87}$.The application of this technique into novel efficient non-fullerene systems and low LUMO/HOMO offset heterojunctions might provide new insight in the photophysics of organic solar cells.

\section{Conclusions}

In conclusion, using ultrafast PPP spectroscopy in organic solar cells, we observe a temperature-dependent dissociation of localised CTSs. By applying the Arrhenius model, we are able to extract a single activation energy for this behaviour which can be associated with the binding energy for localised CTSs. We rationalise our findings using a simple model in which the initially formed CTSs can evolve through one of the two separate pathways on the 
sub-ps timescale: (i) formation of localised (bound) CTSs followed by efficient geminate recombination or (ii) formation of delocalised CTSs eventually dissociating into free charge carriers. We did not observe major differences in the activation energy $(90 \pm 50 \mathrm{meV})$ across different material systems, despite varying both the driving energy for charge separation and the D-A blend ratio. Based on a simple electrostatic model, a 90-meV activation energy corresponds to $\mathrm{a} \sim 1 \mathrm{~nm}$ separation between the electron and the hole in the CTS. We believe that this represents a typical value for most polymer-fullerene systems. As the binding energy of localised CTSs is well above the thermal energy under working conditions, we speculate that it is not the activation barrier for CTSs but the number of available localised states at the interface which is important for the device performance. Our results support the current view about the design rules for the next-generation solar cells that include a better donor-acceptor energetic alignment, a higher degree of charge delocalisation, and a reduced density of lowenergy recombination centres in order to achieve high performance OPVs with low voltage loss, high photocurrent and high fill factor.

\section{Acknowledgements}

We thank Tom Hopper for proofreading and his comments on the manuscript. J. Z. thanks the China Scholarship Council for a PhD scholarship. A.C.J. thanks the Winton Programme for the Physics of Sustainability, the Engineering and Physical Sciences Research Council, and the University of Cambridge (CHESS) for funding. A.A.B. is a Royal Society University Research Fellow. D.D. acknowledges St John's College, Cambridge for support.

\section{Table of Content Image}

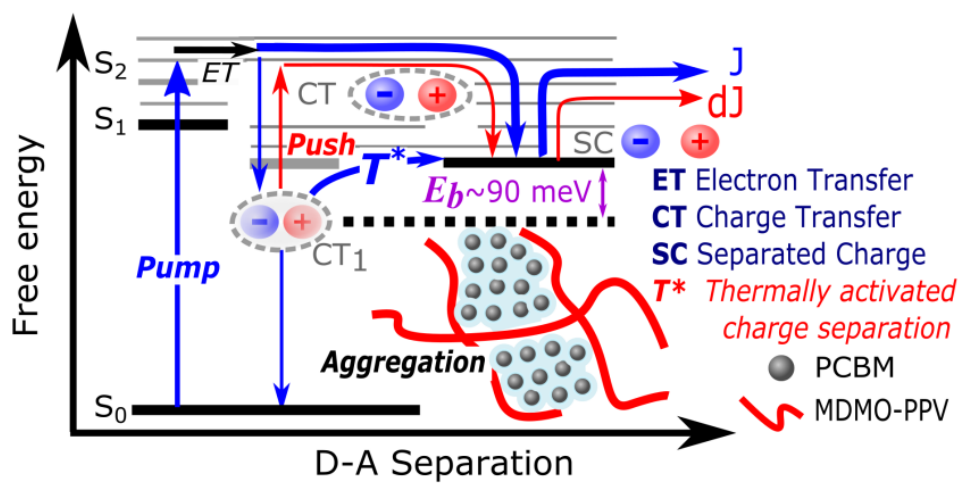




\section{Reference}

$1 \quad$ G. Yu, J. Gao, J. C. Hummelen, F. Wudl and A. J. Heeger, Science, 1995, 270, 17891791.

2 J. J. M. Halls, C. A. Walsh, N. C. Greenham, E. A. Marseglia, R. H. Friend, S. C. Moratti and A. B. Holmes, Nature, 1995, 376, 498-500.

3 C. J. Brabec, G. Zerza, G. Cerullo, S. De Silvestri, S. Luzzati, J. C. Hummelen and S. Sariciftci, Chem. Phys. Lett., 2001, 340, 232-236.

4 A. A. Bakulin, J. C. Hummelen, M. S. Pshenichnikov and P. H. M. van Loosdrecht, Adv. Funct. Mater., 2010, 20, 1653-1660.

5 T. M. Clarke and J. R. Durrant, Chem. Rev., 2010, 110, 6736-6767.

6 J. L. Brédas, J. E. Norton, J. Cornil and V. Coropceanu, Acc. Chem. Res., 2009, 42, 1691-1699.

7 F. Gao and O. Inganäs, Phys. Chem. Chem. Phys., 2014, 16, 20291-304.

8 K. Vandewal, Annu. Rev. Phys. Chem., 2016, 67, 113-133.

9 H. Bässler and A. Köhler, Phys. Chem. Chem. Phys., 2015, 17, 28451-62.

10 X. Zhu, N. R. Monahan, Z. Gong, H. Zhu, K. W. Williams and C. A. Nelson, J. Am. Chem. Soc., 2015, 137, 8313-20.

11 G. J. Hedley, A. Ruseckas and I. D. W. Samuel, Chem. Rev., 2017, 117, 796-837.

12 F. Laquai, D. Andrienko, C. Deibel and D. Neher, in Elementary Processes in Organic Photovoltaics, ed. K. Leo, Springer International Publishing, Cham, 2017, pp. 267-291.

13 M. Antonietta Loi and A. Troisi, Phys. Chem. Chem. Phys., 2014, 16, 20277-20278.

14 J. D. Servaites, M. A. Ratner and T. J. Marks, Energy Environ. Sci., 2011, 4, 44104422 .

15 B. M. Savoie, A. Rao, A. A. Bakulin, S. Gelinas, B. Movaghar, R. H. Friend, T. J. Marks and M. A. Ratner, J. Am. Chem. Soc., 2014, 136, 2876-84.

16 B. Bernardo, D. Cheyns, B. Verreet, R. D. Schaller, B. P. Rand and N. C. Giebink, Nat. Commun., 2014, 5, 3245. 
17 G. D’Avino, L. Muccioli, Y. Olivier and D. Beljonne, J. Phys. Chem. Lett., 2016, 7, $536-540$.

18 S. Gelinas, A. Rao, A. Kumar, S. L. Smith, a. W. Chin, J. Clark, T. S. van der Poll, G.

C. Bazan and R. H. Friend, Science, 2014, 343, 512-516.

19 G. Nan, X. Zhang and G. Lu, J. Phys. Chem. C, 2015, 119, 15028-15035.

20 S. Shoaee, S. Subramaniyan, H. Xin, C. Keiderling, P. S. Tuladhar, F. Jamieson, S. A. Jenekhe and J. R. Durrant, Adv. Funct. Mater., 2013, 23, 3286-3298.

21 A. A. Bakulin, A. Rao, V. G. Pavelyev, P. H. M. van Loosdrecht, M. S. Pshenichnikov, D. Niedzialek, J. Cornil, D. Beljonne and R. H. Friend, Science, 2012, 335, 1340-1344.

22 G. Grancini, M. Maiuri, D. Fazzi, A. Petrozza, H.-J. Egelhaaf, D. Brida, G. Cerullo and G. Lanzani, Nat. Mater., 2013, 12, 29-33.

23 A. C. Jakowetz, M. L. Bohm, A. Sadhanala, S. Huettner, A. Rao and R. H. Friend, Nat Mater, 2017, 16, 551-557.

24 P. E. Hartnett, C. M. Mauck, M. A. Harris, R. M. Young, Y.-L. Wu, T. J. Marks and M. R. Wasielewski, J. Am. Chem. Soc., 2017, 139, 749-756.

25 F. Gao, W. Tress, J. Wang and O. Inganäs, Phys. Rev. Lett., 2015, 114, 128701.

26 B. A. Gregg, J. Phys. Chem. Lett., 2011, 2, 3013-3015.

27 N. R. Monahan, K. W. Williams, B. Kumar, C. Nuckolls and X.-Y. Zhu, Phys. Rev. Lett., 2015, 114, 247003.

28 D. Amarasinghe Vithanage, A. Devižis, V. Abramavičius, Y. Infahsaeng, D. Abramavičius, R. C. I. MacKenzie, P. E. Keivanidis, A. Yartsev, D. Hertel, J. Nelson, V. Sundström and V. Gulbinas, Nat. Commun., 2013, 4, 2334.

29 S. N. Hood and I. Kassal, J. Phys. Chem. Lett., 2016, 7, 4495-4500.

30 S. H. Park, A. Roy, S. Beaupré, S. Cho, N. Coates, J. S. Moon, D. Moses, M. Leclerc, K. Lee and A. J. Heeger, Nat. Photonics, 2009, 3, 297-302.

31 J. Lee, K. Vandewal, S. R. Yost, M. E. Bahlke, L. Goris, M. A. Baldo, J. V Manca and T. Van Voorhis, J. Am. Chem. Soc., 2010, 132, 11878-80.

32 K. Vandewal, S. Albrecht, E. T. Hoke, K. R. Graham, J. Widmer, J. D. Douglas, M. 
Schubert, W. R. Mateker, J. T. Bloking, G. F. Burkhard, A. Sellinger, J. M. Frechet, A. Amassian, M. K. Riede, M. D. McGehee, D. Neher and A. Salleo, Nat Mater, 2014, 13, $63-68$.

33 P. A. Lane, P. D. Cunningham, J. S. Melinger, O. Esenturk and E. J. Heilweil, Nat. Commun., 2015, 6, 7558.

34 K. Chen, A. J. Barker, M. E. Reish, K. C. Gordon and J. M. Hodgkiss, J. Am. Chem. Soc., 2013, 135, 18502-12.

35 H. Hoppe, T. Glatzel, M. Niggemann, W. Schwinger, F. Schaeffler, A. Hinsch, M. C. Lux-Steiner and N. S. Sariciftci, Thin Solid Films, 2006, 511-512, 587-592.

36 T. L. Benanti and D. Venkataraman, Photosynth. Res., 2006, 87, 73-81.

37 J. M. Frost, F. Cheynis, S. M. Tuladhar and J. Nelson, Nano Lett., 2006, 6, 1674-1681.

38 Q. Zhang, B. Kan, F. Liu, G. Long, X. Wan, X. Chen, Y. Zuo, W. Ni, H. Zhang, M. Li, Z. Hu, F. Huang, Y. Cao, Z. Liang, M. Zhang, T. P. Russell and Y. Chen, Nat. Photonics, 2014, 9, 35-41.

39 S. Günes, H. Neugebauer and N. S. Sariciftci, Chem. Rev., 2007, 107, 1324-1338.

40 Y. Liu, J. Zhao, Z. Li, C. Mu, W. Ma, H. Hu, K. Jiang, H. Lin, H. Ade and H. Yan, Nat. Commun., 2014, 5, 5293.

41 D. Deng, Y. Zhang, J. Zhang, Z. Wang, L. Zhu, J. Fang, B. Xia, Z. Wang, K. Lu, W. Ma and Z. Wei, Nat. Commun., 2016, 7, 13740.

42 Z. Guan, H.-W. Li, Y. Cheng, Q. Yang, M.-F. Lo, T.-W. Ng, S.-W. Tsang and C.-S. Lee, J. Phys. Chem. C, 2016, 120, 14059-14068.

43 C. Piliego and M. A. Loi, J. Mater. Chem., 2012, 22, 4141.

44 K. Vandewal, K. Tvingstedt, A. Gadisa, O. Inganäs and J. V. Manca, Phys. Rev. B, $2010, \mathbf{8 1}, 125204$.

45 G. Nan, X. Zhang and G. Lu, Phys. Chem. Chem. Phys., 2016, 18, 17546-17556.

46 C. Deibel, T. Strobel and V. Dyakonov, Adv. Mater., 2010, 22, 4097-4111.

47 I. G. Scheblykin, A. Yartsev, T. Pullerits, V. Gulbinas and V. Sundström, J. Phys. Chem. B, 2007, 111, 6303-6321. 
48 T. M. Clarke, J. Peet, C. Lungenschmied, N. Drolet, X. Lu, B. M. Ocko, A. J. Mozer and M. A. Loi, J. Mater. Chem. A, 2014, 2, 12583.

49 S. M. Menke, A. Sadhanala, M. Nikolka, N. A. Ran, M. K. Ravva, S. Abdel-Azeim, H. L. Stern, M. Wang, H. Sirringhaus, T.-Q. Nguyen, J.-L. Brédas, G. C. Bazan and R. H. Friend, ACS Nano, 2016, 10, 10736-10744.

50 M. Manca, C. Piliego, E. Wang, M. R. Andersson, A. Mura and M. A. Loi, J. Mater. Chem. A, 2013, 1, 7321.

51 P. C. Y. Chow, S. Albert-Seifried, S. Gélinas and R. H. Friend, Adv. Mater., 2014, 26, $4851-4854$.

52 Y. Zou and R. J. Holmes, ACS Appl. Mater. Interfaces, 2015, 7, 18306-18311.

53 S. Leblebici, J. Lee, A. Weber-Bargioni and B. Ma, J. Phys. Chem. C, 2017, 121, $3279-3285$.

54 J. Kern, S. Schwab, C. Deibel and V. Dyakonov, Phys. Status Solidi-Rapid Res. Lett., $2011,5,364-366$.

55 S. Gélinas, O. Paré-Labrosse, C.-N. Brosseau, S. Albert-Seifried, C. R. McNeill, K. R. Kirov, I. A. Howard, R. Leonelli, R. H. Friend and C. Silva, J. Phys. Chem. C, 2011, 115, 7114-7119.

56 M. Muntwiler, Q. Yang, W. A. Tisdale and X. Y. Zhu, Phys. Rev. Lett., 2008, 101, 196403.

57 A. A. Bakulin, Y. Xia, H. J. Bakker, O. Inganäs and F. Gao, J. Phys. Chem. C, 2016, 120, 4219-4226.

58 M.-J. Sher, J. A. Bartelt, T. M. Burke, A. Salleo, M. D. McGehee and A. M. Lindenberg, Adv. Electron. Mater., 2016, 2, 1500351-na.

59 M. Gerhard, A. P. Arndt, I. A. Howard, A. Rahimi-Iman, U. Lemmer and M. Koch, J. Phys. Chem. C, 2015, 119, 28309-28318.

60 A. P. Arndt, M. Gerhard, A. Quintilla, I. A. Howard, M. Koch and U. Lemmer, J. Phys. Chem. C, 2015, 119, 13516-13523.

61 N. Christ, S. W. Kettlitz, S. Valouch, J. Mescher, M. Nintz and U. Lemmer, Org. Electron., 2013, 14, 973-978. 
62 W. J. Grzegorczyk, T. J. Savenije, T. E. Dykstra, J. Piris, J. M. Schins and L. D. A. Siebbeles, J. Phys. Chem. C, 2010, 114, 5182-5186.

R. D. Pensack and J. B. Asbury, J. Am. Chem. Soc., 2009, 131, 15986-7.

64 S. D. Dimitrov, A. A. Bakulin, C. B. Nielsen, B. C. Schroeder, J. Du, H. Bronstein, I. McCulloch, R. H. Friend and J. R. Durrant, J. Am. Chem. Soc., 2012, 134, 18189-92.

65 D. Jarzab, F. Cordella, J. Gao, M. Scharber, H.-J. Egelhaaf and M. A. Loi, Adv. Energy Mater., 2011, 1, 604-609.

66 I. Riedel, J. Parisi, V. Dyakonov, L. Lutsen, D. Vanderzande and J. C. C. Hummelen, Adv. Funct. Mater., 2004, 14, 38-44.

67 A. Petersen, A. Ojala, T. Kirchartz, T. A. Wagner, F. Würthner and U. Rau, Phys. Rev. $B, 2012, \mathbf{8 5}, 245208$.

68 E. A. Katz, D. Faiman, S. M. Tuladhar, J. M. Kroon, M. M. Wienk, T. Fromherz, F. Padinger, C. J. Brabec and N. S. Sariciftci, J. Appl. Phys., 2001, 90, 5343.

69 V. D. Mihailetchi, L. J. A. Koster, J. C. Hummelen and P. W. M. Blom, Phys. Rev. Lett., 2004, 93, 216601.

70 K. Chen, J. K. Gallaher, A. J. Barker and J. M. Hodgkiss, J. Phys. Chem. Lett., 2014, 5, $1732-1737$.

71 M. Gerhard, A. P. Arndt, M. Bilal, U. Lemmer, M. Koch and I. A. Howard, Phys. Rev. $B, 2017,95,195301$.

72 A. J. Barker, K. Chen and J. M. Hodgkiss, J. Am. Chem. Soc., 2014, 136, 12018-26.

73 S. Chen, S.-W. Tsang, T.-H. Lai, J. R. Reynolds and F. So, Adv. Mater., 2014, 26, 6125-6131.

74 H. Hoppe, M. Niggemann, C. Winder, J. Kraut, R. Hiesgen, A. Hinsch, D. Meissner and N. S. Sariciftci, Adv. Funct. Mater., 2004, 14, 1005-1011.

75 R. A. Marsh, J. M. Hodgkiss and R. H. Friend, Adv. Mater., 2010, 22, 3672-3676.

76 A. C. Jakowetz, M. L. Böhm, J. Zhang, A. Sadhanala, S. Huettner, A. A. Bakulin, A. Rao and R. H. Friend, J. Am. Chem. Soc., 2016, 138, 11672-11679.

77 F. Provencher, N. Bérubé, A. W. Parker, G. M. Greetham, M. Towrie, C. Hellmann, M. 
Côté, N. Stingelin, C. Silva and S. C. Hayes, Nat. Commun., 2014, 5, 4288.

78 M. Causa', J. De Jonghe-Risse, M. Scarongella, J. C. Brauer, E. Buchaca-Domingo, J.E. Moser, N. Stingelin and N. Banerji, Nat. Commun., 2016, 7, 12556.

79 L. G. Kaake, C. Zhong, J. A. Love, I. Nagao, G. C. Bazan, T.-Q. Nguyen, F. Huang, Y. Cao, D. Moses and A. J. Heeger, J. Phys. Chem. Lett., 2014, 5, 2000-6.

80 A. E. Jailaubekov, A. P. Willard, J. R. Tritsch, W.-L. Chan, N. Sai, R. Gearba, L. G. Kaake, K. J. Williams, K. Leung, P. J. Rossky and X.-Y. Zhu, Nat. Mater., 2013, 12, $66-73$.

81 A. A. Bakulin, S. D. Dimitrov, A. Rao, P. C. Y. Chow, C. B. Nielsen, B. C. Schroeder, I. McCulloch, H. J. Bakker, J. R. Durrant and R. H. Friend, J. Phys. Chem. Lett., 2013, 4, 209-215.

82 F. Fernández-Lázaro, N. Zink-Lorre and Á. Sastre-Santos, J. Mater. Chem. A, 2016, 4, 9336-9346.

83 C. B. Nielsen, S. Holliday, H.-Y. Chen, S. J. Cryer and I. McCulloch, Acc. Chem. Res., 2015, 48, 2803-12.

84 W. Zhao, D. Qian, S. Zhang, S. Li, O. Inganäs, F. Gao and J. Hou, Adv. Mater., 2016, 28, 4734-4739.

85 J. Liu, S. Chen, D. Qian, B. Gautam, G. Yang, J. Zhao, J. Bergqvist, F. Zhang, W. Ma, H. Ade, O. Inganäs, K. Gundogdu, F. Gao and H. Yan, Nat. Energy, 2016, 1, 16089.

86 N. Liang, W. Jiang, J. Hou and Z. Wang, Mater. Chem. Front., 2017.

87 B. Kan, H. Feng, X. Wan, F. Liu, X. Ke, Y. Wang, Y. Wang, H. Zhang, C. Li, J. Hou and Y. Chen, J. Am. Chem. Soc., 2017, 139, 4929-4934. 